\title{
Thermo-mechanical analysis on thermal deformation of thin stainless steel in laser micro-welding
}

\begin{abstract}
In the present study, a three-dimensional finite element model has been developed to simulate the temperature, stress and deformation fields in continuous wave $(\mathrm{CW})$ laser micro-welding of thin stainless steel sheet. The welding deformation was experimentally evaluated using a single-mode fiber laser with a high-speed scanning system. Application of developed thermal model demonstrated that the laser parameters, such as laser power, scanning velocity and spot diameter have a significant effect on temperature field and the weld pool. In the case of welding deformation, numerical simulation was carried out by an uncoupled thermomechanical model. The welding stress and deformation are generated by plastic deformation during the heating and cooling periods. It was confirmed that the residual stress is higher than yield strength and has strongest effect upon the welding deformation. The numerical simulated results have proved that the developed finite element model is effective to predict thermal histories, thermally induced stresses and welding deformations in the thin material.
\end{abstract}

Keyword: Finite element method; High-speed scanning; Micro-welding; Single-mode fiber laser; Thermal deformation 